\title{
AN APPLICATION OF INTERPOLATING GAIN-SCHEDULING CONTROL
}

\author{
J. De Caigny * J. F. Camino** B. Paijmans * J. Swevers *
}

\author{
${ }^{*}$ Department of Mechanical Engineering \\ Katholieke Universiteit Leuven, Heverlee, Belgium \\ ${ }^{* *}$ School of Mechanical Engineering \\ State University of Campinas, SP, Brazil
}

\begin{abstract}
This work investigates an application of interpolating gain-scheduling control for a structural acoustic problem. The dynamics of the system under consideration are highly sensitive to variation in the temperature. Therefore, linear time invariant $\mathcal{H}_{2}$ output feedback controllers are designed for different temperature conditions. Afterwards, these controllers are interpolated to provide a global discrete-time linear parametervarying controller. The closed-loop stability is a posteriori guaranteed using recent less conservative analyses that consider bounds on the rate of variation of the temperature. Copyright (c) 2007 IFAC.
\end{abstract}

Keywords: active noise control, time varying system, parametric variation, discrete-time.

\section{INTRODUCTION}

A main source of noise within aircraft cabins is the noise generated by the vibration of the surrounding structure, usually denoted as structural noise. In recent years, a significant amount of research in the area of acoustics has been carried out, showing that active control strategies are efficient in reducing noise in the low frequency range (Donadon et al., 2006). Several of these techniques assume that the plant under consideration is linear and time invariant, although, for some applications, this is not a realistic assumption. For instance, the structure is frequently subject to temperature changes, and consequently, its dynamics change considerably according to the temperature.

To deal with a time-varying system, the nominal model and the uncertainty bounds should be appropriately determined, since the trade-off between performance and robustness plays an important role in the control design. For most practical applications, this is a difficult task, and the final estimated uncertainty set is in general too conservative. Therefore, a more elaborate strategy should be applied. Two distinct ap- proaches are commonly used to control linear time varying systems, interpolating gain-scheduling (IGS) control and linear parameter-varying (LPV) control.

The main idea of the IGS control design is to split the control design into two parts. First, local controllers are designed for linearized models of the plant computed at several fixed values of the varying parameter. Second, a global parameter-dependent controller is obtained by scheduling or interpolating these local controllers. Although the stability of the closedloop system cannot be guaranteed, this approach has been successfully used in many industrial applications (Nichols et al., 1993; Aouf et al., 2002).

On the other hand, the LPV control design framework, developed earlier in the 90's, guarantees asymptotic stability of the closed-loop system. However, the existing techniques for synthesis and analysis are still significantly conservative for practical applications as pointed out in (Wassink et al., 2005). Moreover, they require an LPV model of the system which is difficult to obtain from measured data since LPV identification is still in a state of development. The variety of available LPV design strategies is also limited when 
compared to the IGS technique, which can use any well-established linear control design strategy. For the case of $\mathcal{H}_{2}$ dynamic output feedback control design for discrete-time LPV systems, to the best of our knowledge, the only available technique is the one presented in (Apkarian et al., 2000). A survey on gain scheduling for nonlinear control design can be found in (Rugh and Shamma, 2000; Leith and Leithead, 2002).

The interpolating gain-scheduling (IGS) controller presented in Section 2 is an elegant solution for the control of LPV systems. However, stability for all physically possible parameter trajectories is not guaranteed. Therefore, it is necessary to check the stability of the closed-loop system after designing the IGS controller. This is analyzed in Section 3. By combining the interpolating methodology with an a posteriori closed-loop stability certificate, the IGS design becomes a practical engineering tool, as shown in Section 4 for a vibroacoustic application.

\section{THE INTERPOLATING METHODOLOGY}

This section presents the interpolating technique used to construct a parameter-dependent model given a set of linear time invariant (LTI) models selected at different operating conditions. Here, we do not distinguish between plant and controller, since this technique can be used to interpolate either models. Thus, it can be used to estimate an LPV model by interpolating local plants, or as a gain-scheduling control technique by interpolating local controllers designed for fixed operating conditions. The presented approach can be applied for both continuous- and discrete- time systems.

Different methods to interpolate local models exits. A straightforward approach is to interpolate the entries of the local state-space system matrices, assuming the same canonical state-space representation is used. For instance, the authors in (Wassink et al., 2005) used the controllable form. However, as indicated by the authors, this can lead to numerically ill-conditioned LPV models, reducing the use of this method for higherorder systems. Our interpolation technique does not explicitly interpolate the entries of the system matrices, instead, the interpolation is performed by fitting the poles and zeros of the LPV model on the poles and zeros of the local models. The resulting model is a well-conditioned parameter-dependent state-space model. A similar, but not equivalent approach, that also interpolates poles and zeros is presented in (Nichols et al., 1993). However, the resulting model is a parameter-dependent transfer function.

Details of the proposed interpolating approach are found in (Paijmans et al., 2006). Here, we briefly describe the main ideas. Suppose $m$ SISO local models with the minimal state-space representation corresponding to $m$ distinct operating conditions $\theta_{\ell}$ of the scheduling parameter $\theta$ are given by

$$
H^{\ell}=\left\{\begin{aligned}
x_{k+1} & =A^{\ell} x_{k}+B^{\ell} u_{k}, \quad \ell=1, \ldots, m \\
y_{k} & =C^{\ell} x_{k}+D^{\ell} u_{k}
\end{aligned}\right.
$$

These local models $H^{\ell}$ are not required to be stable nor minimum-phase. We do assume, however, that each local model $H^{\ell}$ has the same order $n$ and can be decomposed as the system gain times the series connection of first and second order subsystems:

$$
H^{\ell}=G^{\ell} \prod_{\tau=1}^{\tau_{1}+\tau_{2}}\left[\begin{array}{c|c}
A_{\tau}^{\ell} & B_{\tau}^{\ell} \\
\hline C_{\tau}^{\ell} & D_{\tau}^{\ell}
\end{array}\right]
$$

where the symbol $\prod$ should be interpreted as the series connection of state-space models. The scalar $G^{\ell}$ is the system gain, the integer $\tau_{1}$ is the number of first order subsystems associated with the real poles and $\tau_{2}$ is the number of second order subsystems associated with each pair of complex conjugated poles or two real poles. In this division, the subsystems have unit gain, since the system gain is provided by $G^{\ell}$. Throughout the paper, "gain" denotes the gain associated with the zero-pole-gain factorization of the transfer function and not the DC-gain.

Notice that a first order subsystem can have either no zeros or one zero, thus defining two types of first order subsystems. Likewise, three types of second order subsystems can be defined, depending on the number of zeros. We assume that all local models share the same number $\tau_{1}$ of first order subsystems and the same number $\tau_{2}$ of second order subsystems. The local models are also assumed to have the same number of zeros, which are conveniently distributed along the subsystems (with the same ordering for all the models).

Similarly, following the same pattern as the local models, we assume that the desired global LPV model is composed by a system gain times the series connection of $\tau_{1}+\tau_{2}$ affine LPV subsystems

$$
H(\rho(\theta))=G(\rho(\theta)) \prod_{\tau=1}^{\tau_{1}+\tau_{2}}\left[\begin{array}{l|l}
A_{0}^{\tau}+\rho(\theta) A_{1}^{\tau} & B^{\tau} \\
\hline C_{0}^{\tau}+\rho(\theta) C_{1}^{\tau} & D^{\tau}
\end{array}\right]
$$

with

$$
\rho(\theta)=\sum_{i=0}^{N} \rho_{i} \theta^{i}
$$

Since the matrices $B^{\tau}$ and $D^{\tau}$ do not depend on $\rho(\theta)$, the series connection of the subsystems remains affine. We also enforce the LPV subsystems in (1) to have unit gain. Dividing the global LPV model in low-order subsystems allows us to obtain analytical formulas for the poles and zeros of these LPV subsystems. For example, any "unit gain" second order affine LPV subsystem with two poles and two zeros can be put in the following state-space form:

$$
H_{\tau}(\rho(\theta))=\left\{\begin{aligned}
x_{k+1} & =\left(A_{0}^{\tau}+\rho(\theta) A_{1}^{\tau}\right) x_{k}+B^{\tau} u_{k} \\
y_{k} & =\left(C_{0}^{\tau}+\rho(\theta) C_{1}^{\tau}\right) x_{k}+D^{\tau} u_{k}
\end{aligned}\right.
$$

with $B^{\tau}=\left[\begin{array}{ll}1 & 0\end{array}\right]^{T}$ and $D^{\tau}=1$. For this LPV subsystem, the formulas for the poles and zeros are respectively given by 


$$
p^{\tau}(\theta)=\alpha_{1}^{\tau}+\alpha_{2}^{\tau} \rho(\theta) \pm \sqrt{\alpha_{3}^{\tau}+\alpha_{4}^{\tau} \rho(\theta)+\alpha_{5}^{\tau}(\rho(\theta))^{2}}
$$

and

$$
z^{\tau}(\theta)=\beta_{1}^{\tau}+\beta_{2}^{\tau} \rho(\theta) \pm \sqrt{\beta_{3}^{\tau}+\beta_{4}^{\tau} \rho(\theta)+\beta_{5}^{\tau}(\rho(\theta))^{2}}
$$

where $\alpha^{\tau}=\left\{\alpha_{1}^{\tau}, \ldots, \alpha_{5}^{\tau}\right\}$ and $\beta^{\tau}=\left\{\beta_{1}^{\tau}, \ldots, \beta_{5}^{\tau}\right\}$ are invertible functions of the entries of the matrices $A_{0}^{\tau}$, $A_{1}^{\tau}, C_{0}^{\tau}, C_{1}^{\tau}$ in (3). In a similar way, one can derive the formulas for all the others types of subsystems.

The interpolation technique now consists of the following two main steps. First, we determine the $\alpha^{\tau}$, $\beta^{\tau}$ and $\rho_{0}, \rho_{1}, \ldots, \rho_{N}$ by minimizing the least-squares error between the poles and zeros of the local LTI subsystems and the poles and zeros of the affine subsystem, without considering the system gains. For this purpose, we formulate the following nonlinear leastsquares optimization problem:

$$
\begin{aligned}
E=\min _{\alpha, \beta, \rho} \sum_{\tau}^{\tau_{1}+\tau_{2}} \sum_{\ell=1}^{m} \frac{1}{2}\left\|p^{\tau}\left(\theta_{\ell}\right)-\hat{p}_{\ell}^{\tau}\right\|^{2} & \\
& +\frac{1}{2}\left\|z^{\tau}\left(\theta_{\ell}\right)-\hat{z}_{\ell}^{\tau}\right\|^{2},
\end{aligned}
$$

where $\hat{p_{\ell}^{\tau}}$ and $\hat{z_{\ell}^{\tau}}$ denote the poles and zeros of the subsystem $\tau$ for the local model $\ell$. We solve this problem using a standard Levenberg-Marquardt algorithm. Once the optimal $\alpha^{\tau}$ and $\beta^{\tau}$ are found, the unknown entries of the subsystem matrices $A_{0}^{\tau}, A_{1}^{\tau}, C_{0}^{\tau}$ and $C_{1}^{\tau}$ can be directly computed.

Second, after the above optimization is solved, the gain of the entire LPV model has to be determined. For this purpose, we calculate the polynomial

$$
G(\rho(\theta))=\sum_{j=0}^{M} g_{j}(\rho(\theta))^{j}
$$

such that it fits the local gains $G^{\ell}$. Since the $\rho_{i}$ were already determined from (4), it now suffices to solve a linear least square problem to obtain the $g_{j}$. After $G(\rho(\theta))$ is calculated, the complete LPV model is readily obtained as the series connection (1), that is,

$$
H(\rho(\theta))=G(\rho(\theta))\left[\begin{array}{l|l}
A_{0}+\rho(\theta) A_{1} & B \\
\hline C_{0}+\rho(\theta) C_{1} & D
\end{array}\right] .
$$

Note that by choosing $M=0$ in (5), the gain is constant and the LPV model (6) is affine in $\rho(\theta)$.

\section{STABILITY ANALYSES}

Since closed-loop stability is not guaranteed by the IGS technique presented in the previous section, it is necessary to provide a certificate of stability. Different approaches based on the Lyapunov theory exist. The most common ones are based on the quadratic stability (QS) analysis, although they can be very conservative. Trying to reduce this conservatism, techniques using parameter-dependent Lyapunov functions (PDLF) have been recently investigated. However, few results seem to exist that guarantee stability for discrete-time systems with time varying parameters.

In (Xie et al., 1997) a piecewise-constant Lyapunov function is proposed, whereas in (Daafouz and Bernussou, 2001) an affine PDLF is used. These techniques, however, do not explicitly consider the bounds on the rate of variation of the parameters, which can be a source of conservatism. Taking the bounds on the rate of variation explicitly into account, (Haddad and Kapila, 1996) use a modified Lur'e-Postnikov Lyapunov function and (Amato et al., 2005) use a grid over the parameter space that depends on a bound on the rate of variation.

We now shortly present some of the technical details of the works cited above necessary to guarantee closed-loop stability. Consider the following linearparameter varying (LPV) system

$$
x(k+1)=A(\rho(k)) x(k),
$$

where $A(\rho(k))$ belongs to the following polytopic set

$$
\begin{array}{r}
\mathcal{A}=\left\{A: A=\rho(k) A_{1}+(1-\rho(k)) A_{2},\right. \\
0 \leq \rho(k) \leq 1\} .
\end{array}
$$

Note that the matrix $A$ in (1) can be represented in this form by an appropriate definition of $A_{1}, A_{2}$ and $\rho(k)$. With some abuse of notation we use $\rho(k)$ instead of $\rho(\theta(k))$. Usually, the system is considered slowly varying whenever $|\rho(k+1)-\rho(k)|$ is sufficiently small compared to the dynamics of the system.

For system (7) in the polytope given by (8), it is well known that asymptotic stability is guaranteed if there exists a quadratic Lyapunov function

$$
V(x(k), \rho(k))=x(k)^{T} P(\rho(k)) x(k)
$$

with $P(\rho(k))$ a bounded symmetric positive definite matrix for all $k \geq 0$ satisfying

$$
A(\rho(k))^{T} P(\rho(k+1)) A(\rho(k))-P(\rho(k))<0 .
$$

If the matrix $P(\rho(k))$ in (9) is assumed constant, the problem reduces to the standard QS analysis for which it suffices to check the LMI (9) only on the vertices of the polytope given by (8). The QS analysis allows for arbitrary fast variations of the scheduling parameter, and in general, is a conservative approach.

For a time varying matrix $P(\rho(k))$, the results in (Daafouz and Bernussou, 2001), which we denote by DS, alleviate some of the conservatism associated with the standard QS analysis. Using a PDLF that depends on the parameter $\rho(k)$, sufficient conditions are derived for the stability of (7) in the polytope given by (8). Bounds on the rate of variation are not considered in this approach, thus it can be conservative in some situations. On the other hand, the authors in (Amato et al., 2005) provide a sufficient condition for the stability of a linear parameter varying system that considers a bound $\Delta \rho$ on the rate of variation of $\rho(k)$, using a piecewise constant parameter-dependent Lyapunov function. This condition is denoted by AS. 


\section{RESULTS}

This section presents the results obtained by applying the methods proposed in Section 2 and Section 3 to the structural acoustic problem. First, the experimental setup is presented, followed by an overview of the frequency response function (FRFs) measured at different set points of the scheduling parameter $\theta$, in this case the temperature.

Based on the measured FRFs, local plant models are identified and for each of these local models an $\mathcal{H}_{2}$ controller is designed. Then, using the IGS procedure described in Section 2, these local controllers are interpolated to obtain a global LPV controller that is affine in $\rho(\theta)$, where $\rho(\theta) \in[0,1]$ is a polynomial function of the temperature $\theta$ in degrees Celsius.

Since the IGS approach does not guarantee closedloop stability, different methods from the literature, presented in Section 3, are used to check the closedloop stability. For this purpose, the IGS procedure is used to derive an estimated model for the open-loop plant that is affine in $\rho(\theta)$ as well. Using the same polynomial $\rho(\theta)$ for both the affine LPV controller and the affine LPV model results in an affine closedloop that depends on the same $\rho(\theta)$.

\subsection{Experimental data and its estimated model}

The setup consists of a lexan plate, highly sensitive to the temperature, clamped on a rigid baffle. For details see (Donadon et al., 2006). The exogenous disturbance $w$ that causes the vibration of the plate is provided by a point force driven by a shaker. The control input $u$ used to attenuate the sound pressure inside a semi-anechoic room is provided by a flexural moment driven by a piezoelectric patch attached to the plate. The sound pressure measured by a single microphone, located near the plate, provides the measured output $y$. We use $z=y$ as the controlled output. Thus, the system represented by $y=H_{y w} w+H_{y u} u$ has two inputs, $w$ and $u$, and one output $y$.

We experimentally measured the FRFs at four different temperatures $\theta=\{22.9,23.4,24.4,25.4\}$. For each operating condition, we compute an estimate state-space model using the command PEM from the Matlab Identification toolbox, which uses a subspace method and further refines the model by optimizing the prediction error fit. Figure 1(a) presents the magnitude of the experimental FRFs $\hat{H}_{y w}$ and of the estimated model $H_{y w}$, from the disturbance $w$ to the output $y$. Figure 1(b) shows the magnitude of the experimental FRFs $\hat{H}_{y u}$ and of the estimated model $H_{y u}$, from the control input $u$ to the output $y$. All estimated models are 10th order and have the same number of poles and zeros. For this application, we focus on the frequency range $120-260 \mathrm{~Hz}$. The sampling frequency is $f_{s}=2048 \mathrm{~Hz}$.

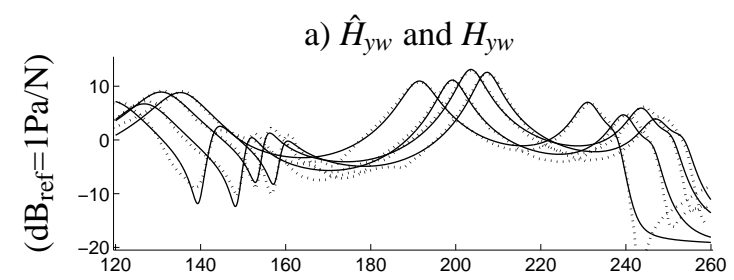

b) $\hat{H}_{y u}$ and $H_{y u}$

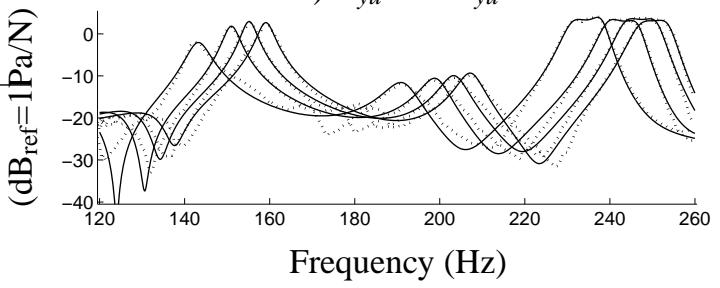

Fig. 1. Measured FRFs $\hat{H}_{y w}$ and $\hat{H}_{y u}$ (dotted lines) and estimated models $H_{y w}$ and $H_{y u}$ (solid lines).

\subsection{Designing the IGS controller}

For each of these 10th-order estimated models, an $\mathcal{H}_{2}$ controller is designed using the same weighting function as in (Donadon et al., 2006). These controllers, shown in Figure 2 (dotted lines), provide satisfactory real-time performance for the corresponding fixed operating condition. On average, they reduced the closed-loop $\mathcal{H}_{2}$ norm by an amount of approximately $35 \%$ compared to the open loop situation. However, we emphasize that the standard $\mathcal{H}_{2}$ controller is not robust (Doyle, 1987).

Each of the local controllers has $n=10$ poles (four complex pole pairs and two real poles), and $n_{z}=9$ zeros (four complex zero pairs and one real zero). Each local controller is decomposed into the product of five second-order subsystems, thus $\tau_{1}=0$ and $\tau_{2}=$ 5 . Note that we could have chosen $\tau_{1}=2$ and $\tau_{2}=4$ as well. The complex zeros are placed in the subsystems containing the complex poles and the real zero in the remaining subsystem.

Using the IGS procedure described in Section 2, an LPV controller $K$, affine in $\rho(\theta)$, will be determined in order to interpolate these local $\mathcal{H}_{2}$ controllers. The LPV controller $K$ will be in the form (6), that is, it will be given by

$$
K(\rho(\theta))=\bar{G}_{K}\left[\begin{array}{c|c}
A_{K 0}+\rho(\theta) A_{K 1} & B_{K} \\
\hline C_{K 0}+\rho(\theta) C_{K 1} & D_{K}
\end{array}\right] .
$$

To proceed, we choose the order $N$ of $\rho(\theta)$ in (2) to be $N=3$ and solve the optimization problem (4) in the unknowns $A_{K 0}, A_{K 1}, B_{K}, C_{K 0}, C_{K 1}, D_{K}$ and $\rho(\theta)$. The optimal solution is

$$
\rho(\theta)=1145-142.2 \theta+5.895 \theta^{2}-0.082 \theta^{3},
$$

which satisfies $0 \leq \rho(\theta) \leq 1$ for $22.9 \leq \theta \leq 25.4$. It now remains to determine the controller gain $\bar{G}_{K}$. For this purpose, we choose $M=0$ in (5), such that $\bar{G}_{K}=g_{0}$ is constant (thus, (10) remains affine in $\rho(\theta)$ ) and solve a linear least square problem to fit $g_{0}$ at the gain of the local $\mathcal{H}_{2}$ controllers. 
Figure 2 shows the affine LPV controller evaluated at ten equidistant points (solid lines) together with the local controllers (dotted lines). Figure 3 shows the variation of two of the ten poles of the controller in the $z$-plane. The diamonds indicate the poles of the local controllers and the $\mathrm{x}$-marks indicate the poles of the LPV controller evaluated at ten different operating conditions. The thick solid line in the top of the graphic represents part of the unit circle and the arrows indicate increasing values of temperature. These figures show that the resulting LPV controller provides a good fit for the local controllers.

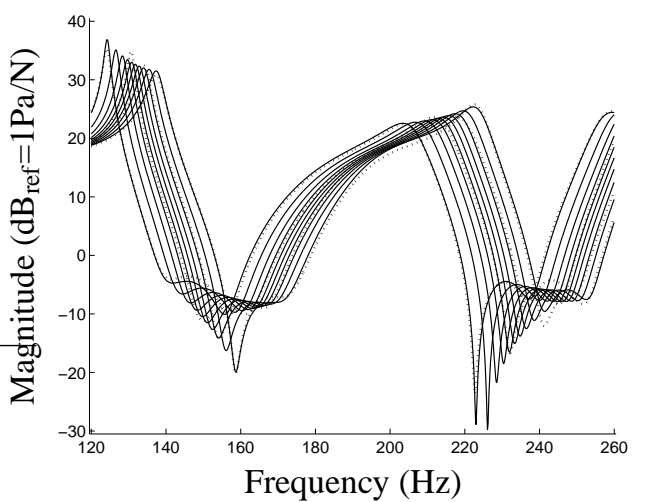

Fig. 2. Local LTI controller (dotted lines) and LPV controller evaluated at ten equidistant values of the scheduling parameter (solid lines).

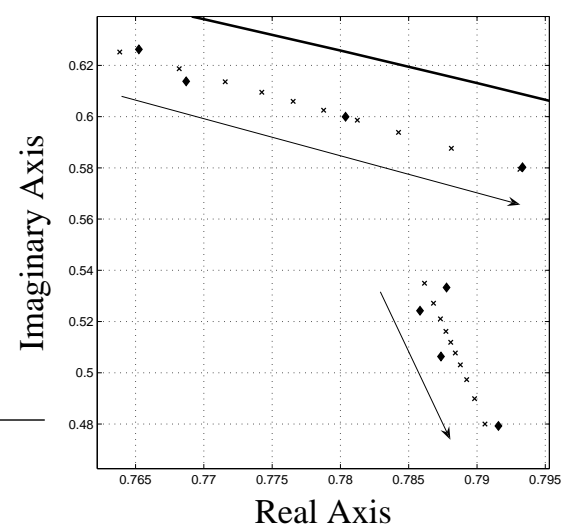

Fig. 3. Variation of two poles for the four local LTI controllers (diamonds) and for ten evaluations of the LPV controller (x-marks).

Recall that the LPV controller evaluated at the four set points is not exactly the same as the LTI controllers specifically designed for these set points. Hence, it is important to check the change in performance that may occur. In this example, the maximum variation of the closed-loop $\mathcal{H}_{2}$ norm is approximately $2.2 \%$, which indicates no significant loss of performance at the set points.

We also evaluate at the four set points two closed-loop systems, one using the local LTI controller (denoted by CL-LTI) and the other one using the LPV controller evaluated at the set points (denoted by CL-LPV). These closed-loop systems for the specific set point $\theta=23.4$ are presented in Figure 4 . The other set points show similar behavior.

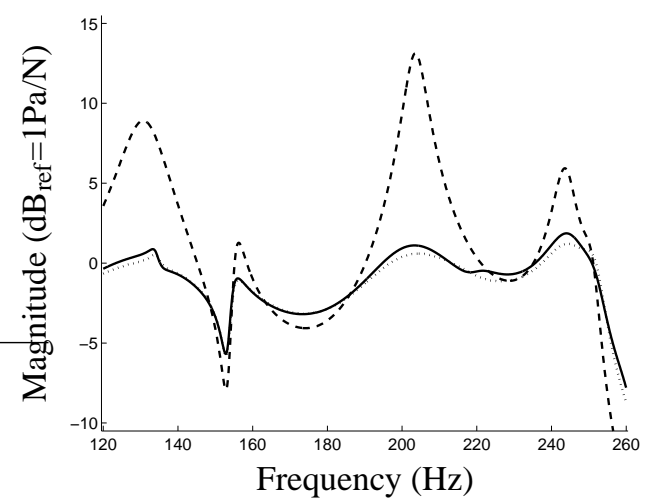

Fig. 4. Open loop $H_{y w}$ (dashed line), closed-loop CLLTI (dotted line), and closed-loop CL-LPV (solid line), computed at $\theta=23.4$.

\subsection{Affine plant interpolation}

To check whether the IGS controller obtained in the previous section ensures stability, we need an LPV model of the closed-loop system. Therefore, an LPV model of the open-loop plant has to be derived. Note that to verify closed-loop stability, we only need to estimate an LPV model for $H_{y u}$.

We impose that the plant model has the same scheduling function $\rho(\theta)$ as the one obtained in the IGS control design given by (11), since this ensures that the resulting closed-loop system will affinely depend on a unique scheduling parameter $\rho(\theta)$. After solving the optimization problem (4), the state-space matrices of the estimated LPV plant model $P(\rho(\theta))$ are readily obtained in the form (6) as

$$
P(\theta)=\bar{G}_{P}\left[\begin{array}{l|l}
A_{P 0}+\rho(\theta) A_{P 1} & B_{P} \\
\hline C_{P 0}+\rho(\theta) C_{P 1} & D_{P}
\end{array}\right],
$$

As for the controller gain, a constant plant gain $\bar{G}_{P}$ is calculated. Figure 5 shows the obtained LPV plant model evaluated at ten equidistant points (solid lines) compared to the local LTI models (dotted lines).

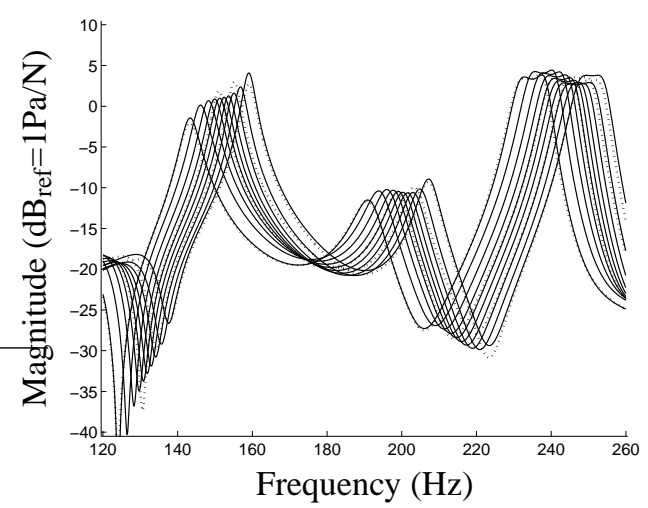

Fig. 5. Local LTI plant (dotted lines) and LPV plant evaluated at ten equidistant values of the scheduling parameter (solid lines). 


\subsection{Closed-loop system analysis}

In this section some of the techniques described in Section 3 are used to check stability of the closed-loop model obtained using the LPV plant $P(\rho(\theta))$ and the LPV controller $K(\rho(\theta))$.

The DS condition that uses an affine parameterdependent quadratic Lyapunov function and the QS condition assume no knowledge on the rate of variation of the temperature. For our example, both conditions were found infeasible, thus being too conservative for our application. The AS condition, on the other hand, incorporates a bound on the rate of variation by limiting the variation of $\rho(\theta(k))$ within one time step to $|\rho(\theta(k+1))-\rho(\theta(k))|<1 / v$. For our application the lowest $v$ was found to be $v=38$ by applying a bisection algorithm. This implies that $\mid \theta(k+1)-$ $\theta(k) \mid<0.0369$, which, for the sampling time used, guarantees closed-loop stability for temperature variations smaller than $75.6^{\circ} \mathrm{C} / \mathrm{s}$.

\section{CONCLUSION}

The proposed interpolating gain-scheduling (IGS) approach is a practical solution for the control of linear time varying systems. Although it does not a priori guarantee closed-loop stability, it possesses some advantages compared to LPV control design techniques. The principal advantage being that the IGS approach uses LTI local controllers that can be designed using well established techniques. For instance, in our application, we used $\mathcal{H}_{2}$ discrete-time local controllers that have satisfactory performance on the real-time setup.

In the literature few techniques have been reported for $\mathcal{H}_{2}$ dynamic output feedback control syntheses for discrete-time LPV models. In general, they are known to be conservative since they are based on quadratic stability conditions. For example the technique proposed in (Apkarian et al., 2000), failed to provide a controller for our application.

The closed loop stability is a posteriori guaranteed using less conservative stability analyses available from the literature. The AS condition allows us to derive an upper bound on the rate of variation of the scheduling parameter. The obtained bound is large enough to allow any realistic variation of temperature.

By combining the IGS approach with an a posteriori stability check, we believe the IGS technique has a large potential to provide satisfactory performance in many practical applications as demonstrated in our noise control problem.

\section{ACKNOWLEDGMENT}

This work is sponsored by the Research Council K.U.Leuven, CoE EF/05/006 Optimization in Engineering (OPTEC), by the Research Foundation Flanders (FWO - Vlaanderen) Proj. G.0446.06, and by the
Belgian Network DYSCO (Dynamical Systems, Control, and Optimization), funded by the Interuniversity Attraction Poles Programme, initiated by the Belgian State, Science Policy Office. The second author has been supported by the Flanders' Mechatronics Technology Centre, Belgium. The authors would like to thank the VibroAcoustic Laboratory of the State University of Campinas were the experimental data were acquired.

\section{REFERENCES}

Amato, F., M. Mattei and A. Pironti (2005). Gain scheduled control for discrete-time systems depending on bounded rate parameters. Int. J. Robust Nonlinear Control 15, 473-494.

Aouf, N., D. G. Bates, I. Postlethwaite and B. Boulet (2002). Scheduling schemes for an integrated flight and propulsion control system. Control Engineering Practice 10(1), 685-696.

Apkarian, P., P. C. Pellanda and H. D. Tuan (2000). Mixed $H_{2} / H_{\infty}$ multi-channel linear parametervarying control in discrete time. Systems \& Control Letters 41, 333-346.

Daafouz, J. and J. Bernussou (2001). Parameter dependent Lyapunov functions for discrete time systems with time varying parametric uncertainties. Systems \& Control Letters 43(5), 355-359.

Donadon, L. V., D. A. Siviero, J. F. Camino and J. R. F. Arruda (2006). Comparing a filtered-X LMS and an $\mathcal{H}_{2}$ controller for the attenuation of the sound radiated by a panel. In: Proc. of the ISMA. Leuven. (cdrom).

Doyle, J. C. (1987). Guaranteed margins for LQG regulators. IEEE Trans. Automat. Contr. 23(4), 756757.

Haddad, W. M. and V. Kapila (1996). Robust stabilization for discrete-time systems with slowly timevarying uncertainty. J. Franklin Inst. 333(1), 7184.

Leith, D. J. and W. E. Leithead (2002). Survey of gainscheduling analysis and design. Int. J. Control 73(11), 1001-1025.

Nichols, R. A., R. T. Reichert and W. J. Rugh (1993). Gain scheduling for H-infinity controllers: A flight control example. IEEE Trans. Contr. Syst. Technol. 1(2), 69-79.

Paijmans, B., W. Symens, H. Van Brussel and J. Swevers (2006). Gain-scheduling control for mechatronic systems with position dependent dynamics. In: Proc. of the ISMA. Leuven. (cdrom).

Rugh, W. J. and J. S. Shamma (2000). Research on gain scheduling. Automatica 36(10), 1401-1425.

Wassink, M. G., M. V. de Wal, C. Scherer and O. Bosgra (2005). LPV control for a wafer stage: beyond the theoretical solution. Control Engineering Practice 13(2), 231-245.

Xie, L., S. Shishkin and M. Fu (1997). Piecewise Lyapunov functions for robust stability of linear time-varying systems. Systems \& Control Letters 31(3), 165-171. 